\title{
Duplicated inferior vena cava - a throwback to embryologic atavism
}

Nandhija Devi $\mathbf{J}^{1}$, Meyyappan $\mathbf{M}^{2}$, Muthiah $\mathbf{B}^{2}$, Rekha $\mathrm{A}^{2^{*}}$.

${ }^{1}$ CRRI,Saveetha Medical College,SIMATS,

${ }^{2}$ Saveetha Medical College, SIMATS

*Corresponding author: A.Rekha, Saveetha Medical College, Saveetha Institute of Medical and Technical sciences (SIMATS)

Received Date: April 28, 2021; Accepted Date: May 03, 2021; Published Date; May 22,2021

Citation: Devi N, Meyyappan J, Muthiah M, Rekha B. (2021) Duplicated inferior vena cava - a throwback to embryologic atavism. J. Surg Case Repo and Imag. 4(4); DOI:10.31579/2690-1897/076

Copyright: (c) 2021 A.Rekha, This is an open-access article distributed under the terms of the Creative Commons Attribution License, which permits unrestricted use, distribution, and reproduction in any medium, provided the original author and source are credited

\begin{abstract}
Duplication of the inferior vena cava( IVC) is only rarely reported and often detected as an incidental finding $(0.5-3 \%)$. In our patient, the anomolous vessel is the continuation of left iliac vein crossing anterior to the aorta, joining the right IVC at the level of inferior border of L1 vertebra. The association with a prothrombotic state,was exacerbated after splenectomy for this patient who had sickle cell anemia.
\end{abstract}

Keyword: inferior vena cava; embryologic atavism

\section{Introduction}

The inferior vena cava is the channel that drains blood from the lower extremities and the abdominal viscera. Its development entails the contribution from the right sub cardinal vein, right supracardinal vein ,right vitelline vein and rightposterior cardinal veins and the anastomoses between these.[1]. Disruption in the regression of their similar counter parts on the left will contribute to a venous anomaly-ivc duplication. The anomalous presentations with respect to ivc may be varied like duplication, agenesis, left sided IVC, continuation with azygos ,hemiazygos.[3,4].

\section{Case report}

A 19 year old boy came to the OPD with complaints of tiredness, diffuse abdominal pain and an episode of fever over a period of 15 days. He gave no history of leg swelling, dyspnoea or palpitations .Clinical examination showed him to anemic and he had a minimal hepatomegaly and a moderate splenomegaly. Routine investigations revealed a $\mathrm{Hb}$ of $5.4 \mathrm{gm} / \mathrm{dl}$ and the peripheral smear delineated microcytic hypochromic RBCs with anisocytosis, few target cell and few sickle cells. The sickling test with sodium metabisulphate corroborated the diagnosis. The confirmation of sickle cell disease (homozygous alleles) was through HPLC.CECT of the abdomen done showed a splenic infarct in an enlarged spleen and minimal hepatomegaly. THE CECT identified the duplication of the IVC, where the left iliac vein continued as the duplicated IVC (Type 2A).

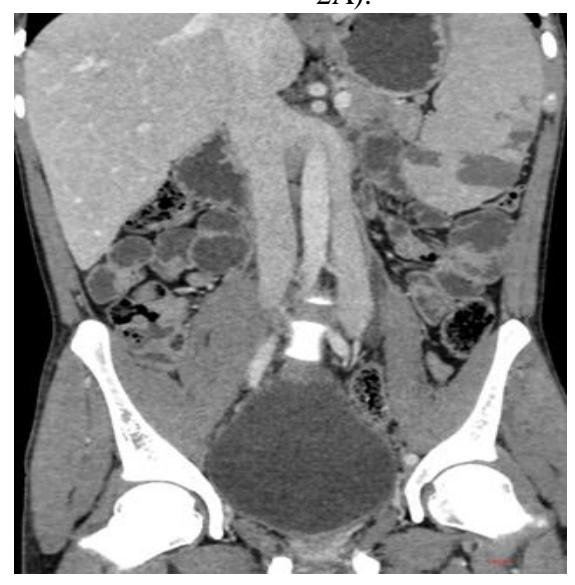

Figure.1-Contrast enhanced CT: coronal view 


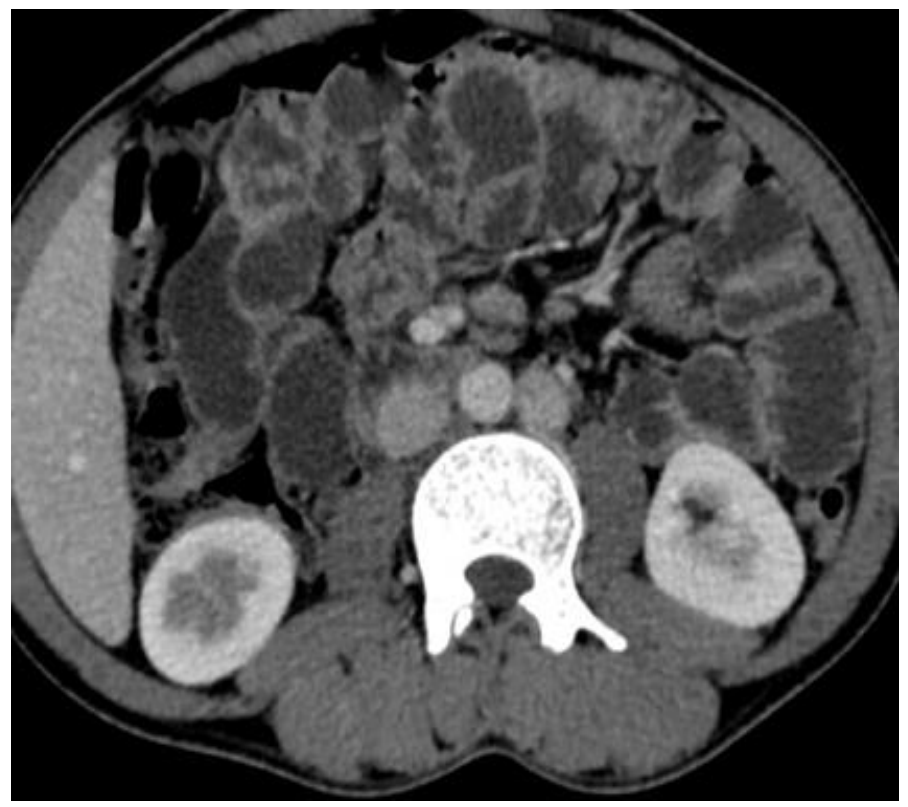

Figure.2-Contrast enhanced CT:axial view

(Fig.1,Fig.2) In our patient there was both right and left IVC and a preaortic segment measuring $1.68 \mathrm{~cm}, 1.57 \mathrm{~cm}$ and $0.47 \mathrm{~cm}$ in diameter respectively.There was no interilliac communication between the vessels and hence it was category 2 a.In view of the splenic infract, with a background of hypersplenism, patient underwent splenectomy after pre op immunisation with polyvalent vaccine .

The prolonged immobilisation following surgery his sickle cell status and post splenectomythrombocytosis (5.2lakhs percubic $\mathrm{mm}$ ) compounded the prothrombotic state that co exists in patients with venous duplication anomalies.He was refered to the vascular department and low dose aspirin was started to prevent venous thrombo embolism.He is well at the 2 month follow up visit.

\section{Discussion}

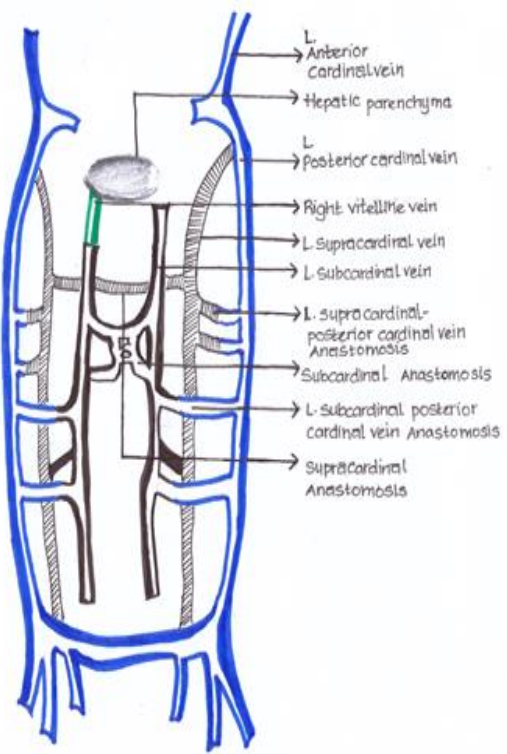

Amongst the various misdirected development involving the IVC, the duplicated IVC is the commonest with an estimated occurrence of about $0.5-3 \%[1$,$] .This aberrant development happens around 5th to the 8$ th week of gestation when the three veins common cardinal vein, the vitelline vein and the umbilical vein on both sides form the capacitance system of the body. The cranial part of the IVC is formed from the right proximal vitelline vein, the rest of the IVC entails contribution from the right sub cardinal, right supra cardinal,the anastomoses between them and the right posterior cardinal vein.[2]. There is no contribution from the left sided system .In essence, the subcardinal vein develops first draining the body wall ,taking over this function from posterior cardinal veins. This involves development of anastomosis channels between the two and is followed by an orderly regression (Fig.3).

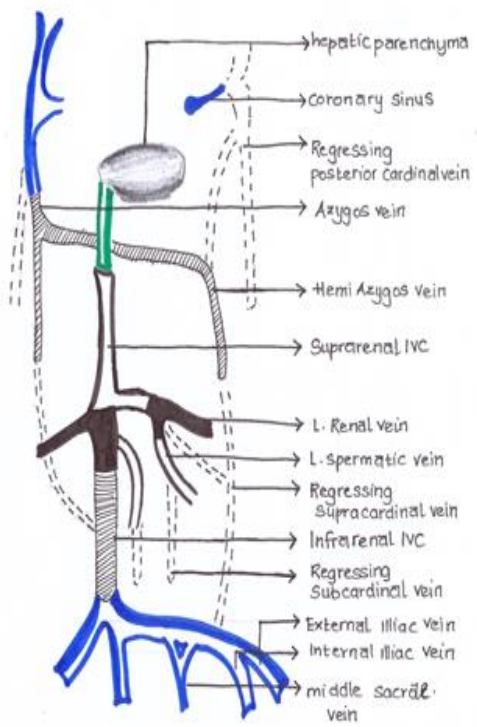


Regression of mostly the anastomotic channel between the two followed later by the posterior cardinal vein. forms the picture of the supra renal portion of IVC .Later on the supra-cardinal veins form relentless anastomosis with posterior cardinal veins, subcardinal veins and with its counterpart on the left.this later would form the infrarenal system and takes over the function of draining the lower half of the body.[3]

The channels on the left mostly regress, with the last to regress being the left supracardinalvein. Persistence of this left supra cardinal vein leads to a duplicated venous system (Fig.4).

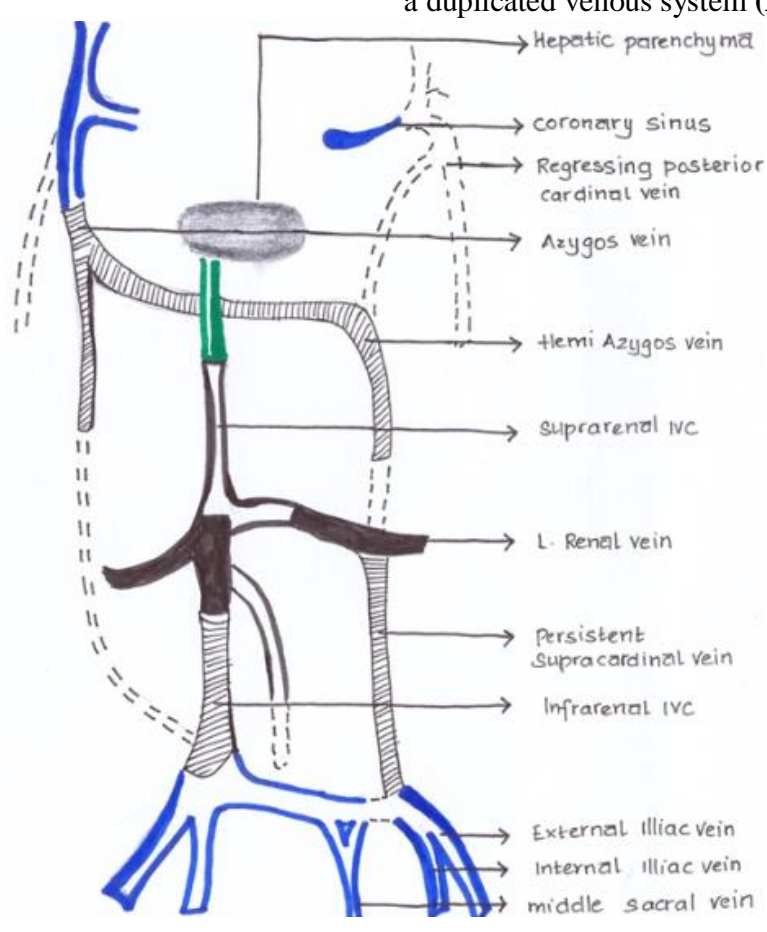

Figure.4-Anomalous development of inferior venocava

Few speculate that the left infrarenal vessels coalesce with the right as opposed to the proposed regression .The Genito urinary systems develops in close association with these vessels in the abdominopelvic region ,the vessels anomalous development deranges their development as well And thus we see associated anomalies like retrocaval ureter, horseshoe kidney. The incidences in various reported age group and gender becomes a very subjective finding for its observance is only incidental but as reported by a study by Morita [4],there is higher incidence of this anomaly in males than in females
There have been studies detailing a classification system for these venous anomalies. The largest study conducted for such classification by Morita had picked up 28 cases with a duplicated IVC. Based on the patterns observed for duplicated IVC alone there was a proposal of 4 types.the study defined an interilliac vein for this classification purpose -the vein that connects iliac veins be it common, internal or external iliac vein to the ivc on the contralateral side.[4]

The classification :(Fig.5)
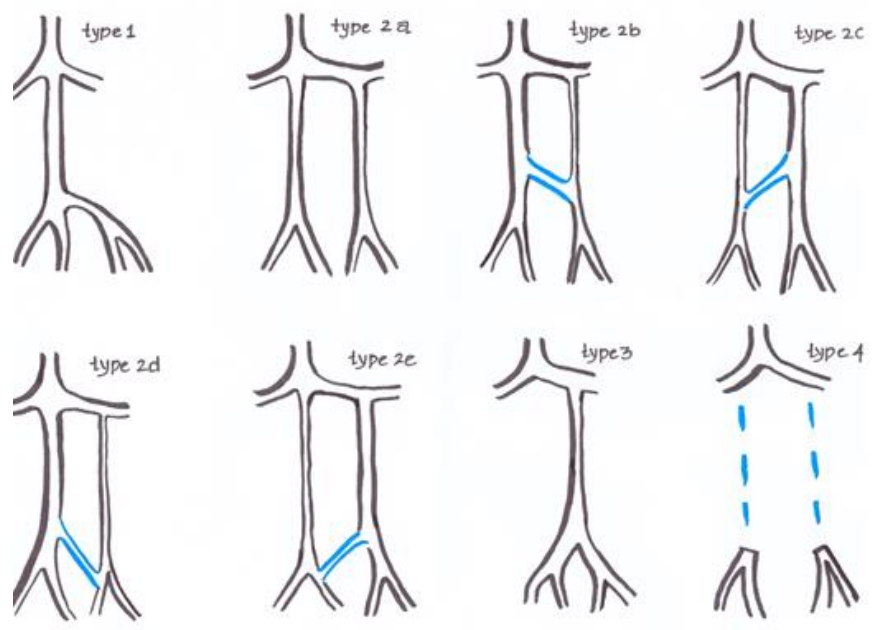
2a -ivc duplication with no inter illiac communication ,2b-ivc duplication with inter illiac vein arising from left common illiac vein,2c -ivc duplication with interilliac vein arising from right common iliac vein, $2 \mathrm{~d}$ ivc duplication with interilliac vein arising from left internal illiacvein,2eivc duplication with inter iliac vein arising from right internal iliac vein.Among all these type $2 \mathrm{a}$ was reported to be most common.

The study also observed a left to right ivc diameter ratio to show that in a case with a high ratio the interilliacvein was draining into the left. Thus naturally the left caliber was greater than the right.

The recent classification system to posed by Natsis[5] was based on the vessels caliber

Type 1 - major duplication, where the left and right IVC are of same caliber with that of preaortic segment.

Type 2 - minor duplication where left and right IVC are of same caliber but are both smaller than the preaortic segment.

Type 3- asymmetrical duplication: with either left or right IVC having larger diameter but both being smaller than the preaortic segment.

With vessels anastomosing in front of aorta and the aorta[6] exerting some pressure there is room for stasis and hyper coagulation as suggested by Gayer. IVC malformation is a potential risk factor for thrombus formation as Virchow's triad is met. Clinically they present with deep vein thrombosis(DVT)[7,9] or pulmonary embolism .[8].

Duplication of IVC thus complicates IVC filter placement], retro peritoneal surgeries, radical nephrectomy of total renal transplant and testicular malignancy radio imaging. They can be wrongly reported as paraaortic lymphadenopathy, aortic aneurysm or retro peritoneal cyst and can mislead the radiologist and the surgeon [10].

Good preoperative imaging with a CECT or MRI is crucial for the diagnosis $[11,12,13,14]$.

CECT has the disadvantage of radiation exposure but it is still a tool of remark for it helps pick up various other pelvic anomalies like retrocaval ureter, horse shoe kidney[15].MRI by the non usage of radiation, the clear distinct visualization of tissues has itself pegged for an ideal tool of imaging.

Contrast enhanced 3D MRV has now been accepted as a reference standard for diagnostic visualization of vasculature. It provides a simplified imaging of the venous system without soft tissue superimposition.

In the study by Hofstaetterthe simple fetalultrasonography done (incorporation of high resolution colour Doppler) in during the embryological period (post development of venous system formationafter 8 weeks) could delineate the defect $[16,17,18]$.

Anecdotal reports are available for the management of the prothrombotic states in these patients with anti coagulation,thrombolysis and IVC filters.[19,20,21,22,23]There is no universally agreed position for filter placement in the scenario of asymptomatic IVC .

\section{Conclusion}

IVC duplication is often an incidental finding on imaging. It acquires significance as it is a prothrombotic state[24] , and it can mislead both the radiologist and surgeon. Given that it is uncommon,we $\mathrm{n}$ eed to rely on anecdotal reports to plan management strategies. A good preoperative pickup in our patient ( sickle cell anemia undergoing splenectomy), enabled us to start early anticoagulation in the post operative period.

\section{References}

1. Pansky, Ben. Review of medical embryology. Macmillan, was.

2. Sadler, Thomas W. Langman's medical embryology. Lippincott Williams \& Wilkins, 2018.

3. Mayo, J., R. Gray, E. St Louis, H. Grosman, M. McLoughlin. (1983)."Anomalies of the inferior vena cava." American Journal of Roentgenology; 140, 2: 339-345.

4. Morita, S., M. Higuchi, N. Saito, and N. Mitsuhashi. (2007)."Pelvic venous variations in patients with congenital inferior vena cava anomalies: classification with computed tomography." ActaRadiologica; 48,9: 974-979.

5. Natsis, Konstantinos, StylianosApostolidis, George Noussios, EfthymiaPapathanasiou. (2010)."Duplication of the inferior vena cava: anatomy, embryology and classification proposal." Anatomical science international; 85, no. 1: 56-60.

6. Polguj, M., M. Majos, M. Topol, A. Majos, and L. Stefańczyk. (2014). "The influence of atherosclerotic abdominal aorta on the shape of duplicated inferior vena cava: its potential clinical implications and vascular complications." Folia morphologica 73,4: 521-526.

7. Kouroukis, Chrisostomos, and Jacques R. Leclerc. (1996). "Pulmonary embolism with duplicated inferior vena cava." Chest; 109:4 1111-1113.

8. Anne, Nirupama, RatnakishorePallapothu, Raymond Holmes, and Moses Degraft Johnson. (2005). "Inferior vena cava duplication and deep venous thrombosis: case report and review of literature." Annals of vascular surgery; 19,5: 740-743.

9. Mathews, Ranjiv, Patricia A. Smith, Elliot K. Fishman. (1999). "Anomalies of the inferior vena cava and renal veins: embryologic and surgical considerations." Urology; 53,5: 873880.

10. Chou, Chen-Te, Albert D. Yang, Yu-Cheng Hong, (2006). "Bilateral retrocaval ureters with IVC duplication." Abdominal imaging; 31, 5: 596-597.

11. Smillie, Richard P., Monisha Shetty, Andrew C. Boyer, Beatrice Madrazo. (2015). "Imaging evaluation of the inferior vena cava." Radiographics; 35,2: 578-592.

12. Kandpal, Harsh, Raju Sharma, Shiva Gamangatti, Deep N. (2008). "Imaging the inferior vena cava: a road less traveled." Radiographics; 28,3: 669-689.

13. Quintero, Juan Carlos, Suzanne Pozuelo, Yolanda Roca, Lidia Mon. (2005). "Congenital anomalies of the inferior vena cava: Cross-sectional imaging findings."

14. Bass, J. E., M. D. Redwine, L. A. Kramer, and J. H. Harris Jr. (1999). "Absence of the infrarenal inferior vena cava with preservation of the suprarenal segment as revealed by CT and MR venography." AJR. American journal of roentgenology; 172, no. 6: 1610-1612.

15. Shaaban, Mohamed Samir. (2016). "Congenital anomalies of the inferior vena cava and iliac veins: a cross-sectional study by multi-detector computed tomography." The Egyptian Journal of Radiology and Nuclear Medicine; 47, no. 3: 883-890

16. Bakry, H., Z. Leibovitz, I. Shapiro, B. Weizman, G. Cohen, L. (2019). "P20. 05: Prenatal diagnosis of inferior vena cava duplication." Ultrasound in Obstetrics \& Gynecology 54: 221221.

17. Vijayaraghavan, S. Boopathy, Vaijayanthi Raja, and T. V. Chitra. (2003). "Interrupted inferior vena cava and left-sided subrenal inferior vena cava: prenatal diagnosis." Journal of 
ultrasound in medicine: official journal of the American Institute of Ultrasound in Medicine 22, no. 7: 747-752.

18. Hofstaetter, C., H. Plath, and M. Hansmann. (2000). "Prenatal diagnosis of abnormalities of the fetal venous system." Ultrasound in Obstetrics and Gynecology; 15,3: 231-241.

19. Davari, Hamid Reza, Seyed Ali Malek-Hosseini, HeshmatollahSalahi, Ali Bahador, et.al (2007). "Management of infrarenal duplicated inferior vena cava during living related kidney transplantation." Transplant International; 20,5: 478479.

20. Sartori, Maria Teresa, Paolo Zampieri, Anto-Luigi Andres, Paolo Prandoni, (2006). "Double vena cava filter insertion in congenital duplicated inferior vena cava: a case report and literature review." Haematologica 91,6_Suppl: ECR30ECR30.

21. Malgor, Rafael Demarchi, Marcone Lima Sobreira, PriscilaNunesBoaventura, Regina Moura, (2008). "Filter placement in duplicated inferior vena cava: case report and review of the literature." J Vasc Bras; 7,2: 167-170.

22. Mano, Akiko, TetsuyaTatsumi, Hiromi Sakai, YukoImoto, TetsuyaNomura,. (2004). "A case of deep venous thrombosis with a double inferior vena cava effectively treated by suprarenal filter implantation." Japanese Heart Journal; 45, no. 6: 1063-1069.

23. Tamizifar, Babak, ParisaSeilani, and Maryam RismankarZadeh. (2013). "Duplication of the inferior vena cava and thrombosis: a rare case." Journal of research in medical sciences: the official journal of Isfahan University of Medical Sciences; 18, no. 10: 911.

24. Sparkenbaugh, E., and R. Pawlinski. (2017). "Prothrombotic aspects of sickle cell disease." Journal of Thrombosis and Haemostasis; 15,7: 1307-1316. 